\title{
Erratum regarding missing Declaration of Competing Interest statements in previously published article
}

Declaration of Competing Interest statement was not included in the published version of the following article that appeared in previous issues of Veterinary and Animal Science.

The appropriate Declaration/Competing Interest statements, provided by the Authors, are included below.
1) Effects of blindfolding and tail bending of Egyptian water buffaloes on their behavioural reactivity and physiological responses to pain induction (Veterinary and Animal Science, 2018; 5C: 23) https://doi. org/10.1016/j.vas.2018.06.001. The authors declare that they have no known competing financial interests or personal relationships that could have appeared to influence the work reported in this paper. 